\title{
The effects of low-volume high-intensity interval training and circuit training on maximal oxygen uptake
}

\author{
Stefan T. Birkett ${ }^{1}\left[\right.$ - Simon Nichols ${ }^{2} \cdot$ Richard Sawrey $^{3} \cdot$ Damien Gleadall-Siddall $^{3} \cdot$ Gordon McGregor $^{4} \cdot$ Lee Ingle $^{3}$
}

Received: 16 November 2018 / Accepted: 15 April 2019 / Published online: 2 May 2019

(c) The Author(s) 2019

\begin{abstract}
Purpose High-intensity interval training (HIIT) and circuit training (CT) are popular methods of exercise, eliciting improvements in cardiorespiratory fitness (CRF). However, direct comparisons of these two training methods are limited. We investigated the effects of HIIT and CT on CRF.

Methods Thirty-nine apparently healthy middle-aged participants [HIIT; mean age: $42.5 \pm 12.3 ; \dot{V} \mathrm{O}_{2 \max } 31.5 \pm 7.1$ $\left(\mathrm{ml} \mathrm{kg}{ }^{-1} \min ^{-1}\right)$; $52 \%$ males; CT; mean age: $41.2 \pm 12.9 ; \dot{V} \mathrm{O}_{2 \max } 31.4 \pm 6.8\left(\mathrm{ml} \mathrm{kg}^{-1} \mathrm{~min}^{-1}\right) ; 57 \%$ males] were randomly allocated to two sessions per week of HIIT or CT over 8 weeks. HIIT performed ten 1-min cycle-ergometry intervals at $>85 \%$ $\mathrm{HR}_{\max }$, separated by ten 1-min intervals of active recovery. The CT group performed up to 40 -min of CT at $60-80 \% \mathrm{HR}_{\max }$. $\mathrm{CRF}$ was measured using maximum oxygen uptake $\left(\dot{V} \mathrm{O}_{2 \max }\right)$, ventilatory anaerobic threshold $\left(\dot{V} \mathrm{O}_{2}\right.$ at $\left.\mathrm{VAT}\right)$ and maximum oxygen pulse $\left(\dot{V} \mathrm{O}_{2} / \mathrm{HR}\right)$.

Results $\dot{V} \mathrm{O}_{2 \max }$ increased by $12 \%$ following HIIT (mean difference $3.9 \mathrm{ml} \mathrm{kg}^{-1} \mathrm{~min}^{-1}$; 95\% CI: $2.8-4.9 ; P<0.001$ ), and $3 \%$ in CT (mean difference $1.0 \mathrm{ml} \mathrm{kg}{ }^{-1} \mathrm{~min}^{-1}$; 95\% CI: -0.4 to $2.0 ; P=0.060$ ). $\dot{V} \mathrm{O}_{2}$ at VAT increased by $16 \%$ following HIIT (mean difference $2.4 \mathrm{ml} \mathrm{kg}{ }^{-1} \mathrm{~min}^{-1}$; 95\% CI: 1.6-3.1; $P<0.001$ ) and $4 \%$ in CT (mean difference $0.7 \mathrm{ml} \mathrm{kg}^{-1} \mathrm{~min}^{-1}$; 95\% CI: -0.1 to $1.4 ; P=0.085$ ). $\dot{V} \mathrm{O}_{2} / \mathrm{HR}$ increased by $11 \%$ following HIIT (mean difference $1.4 \mathrm{ml}^{-1}$ beat $^{-1} ; 95 \% \mathrm{CI}$ : $0.9-2.0$; $P<0.001$ ) and $1 \%$ after CT (mean difference $0.3 \mathrm{ml}^{\text {beat }}{ }^{-1} ; 95 \% \mathrm{CI}:-0.3$ to $0.8 ; P=0.318$ ).
\end{abstract}

Conclusion Our study demonstrated that HIIT led to greater improvements in CRF when compared to CT.

Clinical trial registration ClinicalTrials.gov Identifier: NCT03700671.

Keywords Cardiorespiratory fitness $\cdot$ Fidelity $\cdot$ Maximal oxygen consumption $\cdot \dot{V} \mathrm{O}_{2 \max } \cdot$ Ventilatory anaerobic threshold $\cdot$ HIIT

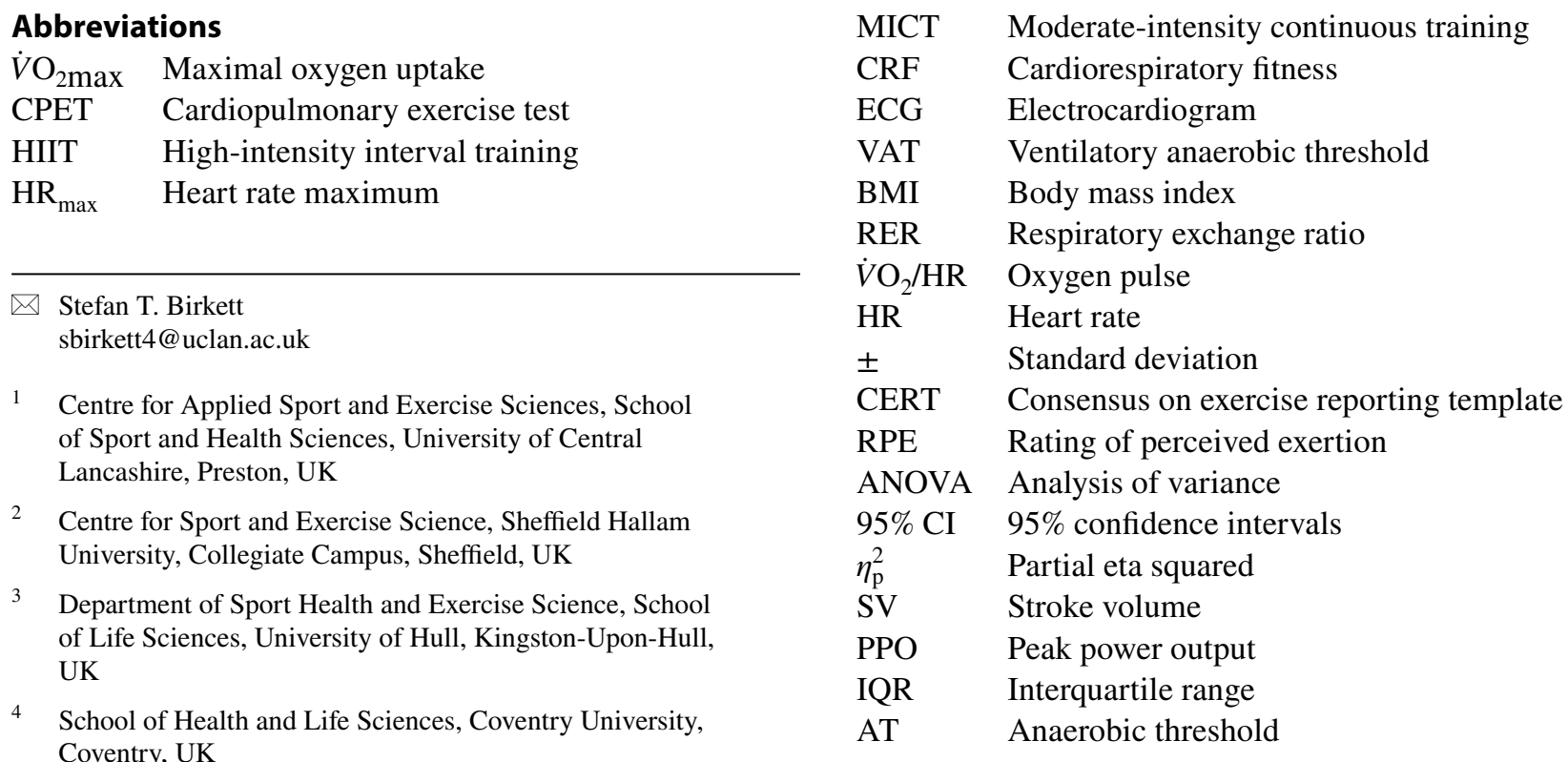




\section{Introduction}

Regular exercise is well known to have a positive effect on health [1, 2]. Many of these benefits are associated with improving maximal oxygen uptake $\left(\dot{V} \mathrm{O}_{2 \max }\right)[3,4]$. Increasing $\dot{V} \mathrm{O}_{2 \text { max }}$ through exercise training may improve cardiometabolic health, quality of life and increase lifeexpectancy $[4,5]$. Popular training methods aimed at improving $\dot{V} \mathrm{O}_{2 \max }$ are circuit and high-intensity interval training (HIIT) and are routinely adopted by the general population, health and fitness professionals and researchers [6]. Circuit training (CT) is typically performed at a moderate or high intensity, over a period of 30-50 min and involves a range of aerobic, body weight and resistance exercises with minimal rest [7, 8]. Low-volume HIIT is defined as 'brief, intermittent bursts of vigorous activity, interspersed by periods of rest or low intensity exercise' [9], typically prescribed at a training intensity between 80 and $100 \%$ of heart rate maximum $\left(\mathrm{HR}_{\max }\right)$ [10].

Whole-body CT and low-volume HIIT consisting of 8-12 1-min interval bouts, interspersed with a similar recovery time have been shown to improve cardiometabolic health and cardiorespiratory fitness (CRF) [8, 11-16]. Increases in $\dot{\mathrm{VO}}_{2 \max }$ and the anaerobic threshold (AT) have been observed following CT; however, published studies are limited to sedentary and older populations, with further markers of CRF not fully explored $[8,17]$. Comparable improvements in $\dot{V} \mathrm{O}_{2 \max }$ and the AT have been shown following low-volume HIIT, with increases in maximum oxygen pulse ( $\left.\dot{V} \mathrm{O}_{2} / \mathrm{HR}\right)$ also observed [16, 18, 19]. However, there is limited evidence directly comparing both training methods across a range of CRF markers, as such it is unknown which approach is most beneficial $[20,21]$.

Direct comparisons have been made investigating lowvolume HIIT and moderate-intensity continuous training (MICT), which is typically 30-60 min in duration adopting the same modality of exercise [14-16]. The results demonstrate low-volume HIIT to be a time-efficient method, eliciting greater improvements in $\dot{V} \mathrm{O}_{2 \max }$, although these findings are not consistently shown [22].

While HIIT and CT are feasible and effective at improving CRF, no study has directly investigated the two. Therefore, the aim of this study was to compare the effect of two weekly sessions of low-volume HIIT and CT over an 8-week period on $\dot{V} \mathrm{O}_{2 \max }$ in apparently healthy middleaged adults. We also investigated changes in other markers of CRF such as the first ventilatory anaerobic threshold ( $\dot{V} \mathrm{O}_{2}$ at VAT) and maximum $\dot{V} \mathrm{O}_{2} / \mathrm{HR}$. Intervention fidelity was also evaluated. We hypothesised that HIIT would elicit superior improvements in $\dot{V} \mathrm{O}_{2 \max }$ and additional markers of CRF.

\section{Methods}

\section{Study design}

Participants were enrolled in a randomised control trial at the University of Hull to either 8 weeks of HIIT or CT (two supervised sessions per week, accompanied by an exercise physiologist). A sample size of 38 using $\mathrm{G}^{*}$ Power 3.1 software was calculated based on previously published data in which the mean difference between HIIT and MICT was $3.2 \mathrm{ml} \mathrm{kg}^{-1} \mathrm{~min}^{-1}$ with a pooled standard deviation of $3 \mathrm{ml} \mathrm{kg}^{-1} \mathrm{~min}^{-1}$ [19]. Statistical significance was set at $\alpha=0.05$ and power set to 0.95 . To allow for $10 \%$ attrition 42 individuals were recruited to the study. To assess the effectiveness of the interventions as determined by $\dot{V} \mathrm{O}_{2 \max }$, a maximal cardiopulmonary exercise test (CPET) to volitional exhaustion on an electronically braked cycle ergometer at baseline (visit one), and following an 8-week exercise intervention of HIIT or CT (visit two) was conducted. When attending the assessments participants were asked not to take part in any strenuous exercise $24 \mathrm{~h}$ prior to the appointment, to wear suitable comfortable clothing and avoid a large meal. For visit two, CPET was performed within 6 days of completing the exercise interventions. A thorough warm-up and cool down were before and after each exercise session. All were asked to maintain their habitual physical activity patterns during the intervention. Body mass index (BMI) was calculated by dividing body weight [23] by height in meters squared and was presented as $\mathrm{kg} \mathrm{m}^{-2}$. Resting blood pressure was measured after 15 min of rest using a sphygmomanometer (A.C. Cossor \& Son Ltd, London UK) and stethoscope (3 M Healthcare, St Paul, MN). To provide a comprehensive account of the study the Consensus on Exercise Reporting Template (CERT) was consulted [24].

\section{Participants}

Ethical approval was provided by the School of Life Sciences ethics committee at the University of Hull which was in accordance with the 1964 Helsinki Declaration and its later amendments or comparable ethical standards. A total of 42 apparently healthy men and women between the age of 18-65 years were recruited to the study. Participant characteristics are given in Table 1. Enrolled individuals reported no medical history of cardiometabolic or limiting respiratory disease, were non-smokers, had a body mass index $<30 \mathrm{~kg} \mathrm{~m}^{-2}$, classified as recreationally active [25] and none were taking any medication that would affect heart rate. A condition of enrolment for those over 45 years was to obtain written medical clearance from a general practitioner and undergo resting and exercise 12-lead electrocardiogram (ECG) (GE Healthcare, Chalfont St Giles, United 
Table 1 Baseline characteristics

\begin{tabular}{lll}
\hline Variable & HIIT $(n=21)$ & $\mathrm{CT}(n=21)$ \\
\hline Males (\%) & 52 & 57 \\
Age (years) & $42.5 \pm 12.3$ & $41.2 \pm 12.9$ \\
$\dot{V} \mathrm{O}_{2 \mathrm{max}}\left(1 \mathrm{~min}^{-1}\right)$ & $2.21 \pm 0.61$ & $2.38 \pm 0.63$ \\
$\dot{V} \mathrm{O}_{2 \mathrm{max}}\left(\mathrm{ml} \mathrm{kg}^{-1} \mathrm{~min}^{-1}\right)$ & $31.5 \pm 7.1$ & $31.4 \pm 6.8$ \\
$\mathrm{VAT}\left(\mathrm{ml} \mathrm{kg}^{-1} \mathrm{~min}^{-1}\right)$ & $15.5 \pm 2.7$ & $15.6 \pm 3.0$ \\
$\mathrm{BMI}\left(\mathrm{kg} \mathrm{m}^{-2}\right)$ & $24.4 \pm 2.3$ & $24.9 \pm 2.7$ \\
Systolic blood pressure $(\mathrm{mmHg})$ & $122 \pm 14$ & $126 \pm 11$ \\
Diastolic blood pressure $(\mathrm{mmHg})$ & $75 \pm 11$ & $79 \pm 9$ \\
Resting HR $(\mathrm{bpm})$ & $68 \pm 12$ & $67 \pm 10$ \\
RER at $\dot{V} \mathrm{O}_{2 \mathrm{max}}$ & $1.30 \pm 0.12$ & $1.31 \pm 0.12$ \\
$\mathrm{HR}$ & $174 \pm 12$ & $174 \pm 14$ \\
Maximum $(\mathrm{bpm})$ & $12.8 \pm 3.7$ & $13.9 \pm 3.9$ \\
Peak power output $(\mathrm{W})$ & $191.9 \pm 43.7$ & $209.8 \pm 55.2$ \\
\hline
\end{tabular}

Values are presented as mean $\pm \mathrm{SD}$. No significant differences between groups at baseline

HIIT low-volume high-intensity interval training, $C T$ circuit training, $\dot{V} \mathrm{O}_{2 \max }$ maximal oxygen consumption, VAT ventilatory anaerobic threshold, $B M I$ body mass index, $H R$ heart rate, $R E R$ respiratory exchange ratio, $H R_{\max }$ maximal heart rate, $\dot{V} \mathrm{O}_{2} / H R$ oxygen pulse

Kingdom). Written informed consent and a pre-exercise medical questionnaire were completed by all.

\section{Cardiopulmonary exercise testing}

Maximal CPETs were conducted in accordance with the American Thoracic Society (ATS) and the American College of Chest Physicians (ACCP) guidelines [26]. An Oxycon pro (Jaeger, Hoechburg, Germany) breath-by-breath metabolic cart was used to collect respiratory gas exchange data. Automatic and manual calibration evaluated ambient temperature, humidity, barometric pressure and altitude. Calibration of the air flow volume was conducted using a 31 syringe and by automatic calibration. Two-point gas calibration was also conducted to ensure accurate measures of inspired $\mathrm{O}_{2}$ and expired $\mathrm{CO}_{2}$ [27]. Tests were performed on a GE e-bike ergometer (GE Healthcare, Buckinghamshire, UK) using a ramp protocol [26]. The protocol consisted of a 3-min rest phase, 3 min of unloaded cycling, followed by a personalised ramp test [28] (ramp rate ranged between 15 and $30 \mathrm{~W}$ ) with work rate continually increased every 1-3 s. Participants performed the same ramp rate pre- and post-testing. Participants were asked to pedal at a cadence of $70 \mathrm{rpm}$ until they reached volitional exhaustion at a protocol duration between 8 and $12 \mathrm{~min}$. Self-reported rating of perceived exertion (RPE) scores using the 6-20 scale [29] and heart rate (HR) (FT1 heart rate monitor, Polar Electro, OY, Finland) was recorded during the last $5 \mathrm{~s}$ of each minute of the test, at maximum exercise and during the recovery period. Together with verbal encouragement to volitional exhaustion, $\dot{V} \mathrm{O}_{2 \max }$ was attained by participants achieving at least two of the following criteria: $\dot{V} \mathrm{O}_{2}$ plateau as determined by a failure of $\dot{V} \mathrm{O}_{2}$ increase by $150 \mathrm{ml} \mathrm{min}^{-1}$ with further increases in workload analysed by breath-by-breath gas exchange data averaged over $15 \mathrm{~s}$ [30], respiratory exchange ratio (RER) $>1.10$, achieve $>85 \%$ age predicated $\mathrm{HR}_{\max }$ and a RPE $>17$ on the 6-20 Borg scale [27]. $\dot{V} \mathrm{O}_{2}$ at VAT was defined using the ' $V$ slope' method [31] and verified using ventilatory equivalents. Peak power output (PPO) (watts) and $\mathrm{HR}_{\max }$ were defined as the highest value achieved during the CPET with maximum $\dot{V} \mathrm{O}_{2} / \mathrm{HR}$ determined by the ratio of $\dot{V} \mathrm{O}_{2 \max }$ and $\mathrm{HR}_{\text {max }}$.

\section{Training interventions}

The HIIT group was asked to perform ten 1-min HIIT intervals, each followed by $1 \mathrm{~min}$ of active recovery (AR) (total exercise time $20 \mathrm{~min}$ ). Resulting from the CPET, HIIT was set at $>85 \% \mathrm{HR}_{\max }$ with a specific HR designated for this criterion. Active recovery was set at a load corresponding to $25-50 \mathrm{~W}$. Sessions were performed on a Wattbike trainer (Wattbike Ltd, Nottingham, UK). The CT group completed a practical seven-station mixed-modality exercise circuit (cycle ergometer, rower, treadmill, sit to stand/squats, knee to elbow and leg kickback with bicep curl) at an intensity of $60-80 \% \mathrm{HR}_{\max }$ (calculated from CPET). No resistance equipment was involved, only body weight. Participants initially performed $20 \mathrm{~min}$ of $\mathrm{CT}$ with duration increased by 5 min per week until the desired $40 \mathrm{~min}$. Each station was occupied for 3-6 minutes depending on session duration, moving from one station to the next with minimal rest. During both interventions, HR was measured in last $5 \mathrm{~s}$ of each station/interval using a FT1 polar heart rate monitor (Polar Electro, OY, Finland) with each CT session timed using a stop watch (Axprod S.L, Guipuzcoa, Spain). Intensity for both interventions was adjusted throughout by the investigator to ensure an appropriate HR range and successful completion of the protocol. Participants were made aware of their HR ranges and verbal encouragement was given by the physiologist to help achieve and maintain these thresholds. Energy expenditure between HIIT and CT was not matched.

To assess the validity of the exercise interventions, participant fidelity to the desired exercise intensity was determined using cut points of $>85 \% \mathrm{HR}_{\max }$ and $60-80 \%$ $\mathrm{HR}_{\max }$ for HIIT and CT, respectively, and reported using previous examples $[32,33]$. These values were calculated using the participants' mean heart rate for each individual interval or station over the 16 sessions and was expressed as a percentage of $\mathrm{HR}_{\max }$ as determined by CPET at visit 1. Specific fidelity thresholds were consulted to determine low $(<50 \%)$, moderate (50-70\%), and high ( $>70 \%)$ compliance [34]. Adherence was determined as a percentage of 
completed sessions, with 14 (>85\%) being the threshold for completion.

\section{Statistical analysis}

Statistical analysis was conducted using SPSS version 24 (IBM, New York, USA). An independent $t$ test was used to identify group differences at baseline. Assumptions of normality were verified using the Shapiro-Wilk test. Skewness and kurtosis of distribution were visually examined. Non-normally distributed data were presented as median and interquartile range (IQR). A two-way (condition $\times$ time) repeated-measures analysis of variance (ANOVA) was used to compare CRF pre-and post-training. Post-hoc analysis for the main effects and interactions was assessed using a Bonferroni adjustment. Group differences were compared using independent $t$ tests. Variables were displayed as mean with 95\% confidence intervals $(95 \% \mathrm{CI})$ or standard deviation $( \pm)$ where specified. Partial eta squared $\left(\eta_{\mathrm{p}}^{2}\right)$ effect sizes were also calculated with $0.01,0.06$ and 0.14 representing small, medium, and large effect sizes, respectively [35].

\section{Results}

Of the 78 CPETs, (pre and post) $95 \%$ of participants achieved a plateau in $\dot{V} \mathrm{O}_{2}$ with all achieving at least two of the desired criteria for maximum effort. Three participants dropped out; one from the HIIT group and two from the CT group (work commitments and an injury unrelated to exercise). The mean training intensities for HIIT were $94 \pm 4 \% \mathrm{HR}_{\max }$ and $76 \pm 3 \% \mathrm{HR}_{\max }$ for CT. The mean exercise duration for the CT group was $32 \pm 4 \min (P<0.001$ versus HIIT) which corresponded to $60 \%$ greater training duration. The proportion of intervals/stations achieving the desired intensity criterion was $90 \%$ (IQR 10\%) for the LVHIIT group and 86\% (IQR 28.5\%) for CT (Fig. 1). Overall adherence for HIIT and CT was $95.2 \%$ and $90.5 \%$, respectively, with 32 participants having $100 \%$ attendance. No adverse events occurred during the exercise sessions.

\section{Low-volume HIIT versus circuit training}

There was a significant main effect for time for $\dot{V} \mathrm{O}_{2 \max }$ (mean difference $2.4 \mathrm{ml} \mathrm{kg}{ }^{-1} \mathrm{~min}^{-1}$; 95\% CI: 1.7-3.2; $\left.P<0.001 ; \eta_{\mathrm{p}}^{2}=0.554\right)$ and interaction effect $(P<0.001$; $\eta_{\mathrm{p}}^{2}=0.302$ ). $V \mathrm{O}_{2 \max }$ significantly increased (Fig. $2 \mathrm{a}$ ) in the HIIT group by $12 \%$ (mean difference $3.9 \mathrm{ml} \mathrm{kg}^{-1} \mathrm{~min}^{-1}$; 95\% CI: $\left.2.8-4.9 ; P<0.001 ; \eta_{\mathrm{p}}^{2}=0.600\right)$ but not in the CT group (3\% increase; mean difference $1.0 \mathrm{ml} \mathrm{kg}^{-1} \mathrm{~min}^{-1} ; 95 \%$ CI: -0.4 to $2.0 ; P=0.060 ; \eta_{\mathrm{p}}^{2}=0.092$ ). HIIT significantly increased $\dot{V} \mathrm{O}_{2 \max }$ to greater extent than CT (mean difference $2.9 \mathrm{ml} \mathrm{kg}^{-1} \mathrm{~min}^{-1}$; $\left.95 \% \mathrm{CI}: 1.4-4.3 ; P<0.001\right)$.
There was a significant main effect for time for $\dot{V} \mathrm{O}_{2}$ at VAT (mean difference $1.5 \mathrm{ml} \mathrm{kg}^{-1} \mathrm{~min}^{-1}$; 95\% CI: 1.0-2.1; $\left.P<0.001 ; \eta_{\mathrm{p}}^{2}=0.474\right)$ and interaction effect $(P<0.05$; $\eta_{\mathrm{p}}^{2}=0.222$ ). $V \mathrm{O}_{2}$ at VAT significantly increased (Fig. $2 \mathrm{~b}$ ) by $16 \%$ after HIIT (mean difference $2.4 \mathrm{ml} \mathrm{kg}^{-1} \mathrm{~min}^{-1}$; 95\% CI: $\left.1.6-3.1 ; P<0.001 ; \eta_{\mathrm{p}}^{2}=0.531\right)$ with no notable changes observed in the CT group (4\% increase; mean difference $0.7 \mathrm{ml} \mathrm{kg}^{-1} \mathrm{~min}^{-1}$; $95 \% \mathrm{CI}$ : -0.1 to $1.4 ; P=0.085$; $\left.\eta_{\mathrm{p}}^{2}=0.078\right)$.

There was a significant main effect for time for maximum $\dot{V} \mathrm{O}_{2} / \mathrm{HR}$ (mean difference $0.8 \mathrm{ml} \mathrm{beat}^{-1}$; 95\% CI: $0.5-1.2$; $\left.P<0.001 ; \eta_{\mathrm{p}}^{2}=0.342\right)$ and interaction effect $(P<0.05$; $\left.\eta_{\mathrm{p}}^{2}=0.189\right)$. Maximum $\dot{V} \mathrm{O}_{2} / \mathrm{HR}$ significantly increased in the HIIT group by $11 \%$ (mean difference $1.4 \mathrm{ml} \mathrm{beat}^{-1}$; 95\% CI: $\left.0.9-2.0 ; P<0.001 ; \eta_{\mathrm{p}}^{2}=0.428\right)$ but not in CT $(1 \%$ increase; mean difference $0.3 \mathrm{ml} \mathrm{beat}^{-1} ; 95 \% \mathrm{CI}:-0.3$ to $\left.0.8 ; P=0.318 ; \eta_{\mathrm{p}}^{2}=0.027\right)$. Both groups increased PPO, HITT by $14 \%$ (mean difference 27 watts; 95\% CI: 19.2-34.3; $P<0.001 ; \eta_{\mathrm{p}}^{2}=0.601$ ) and CT by $6 \%$ (mean difference $\left.11.7 \mathrm{~W} ; 95 \% \mathrm{CI}: 4.2-19.2 ; P<0.01 ; \eta_{\mathrm{p}}^{2}=0.213\right)$. $\mathrm{HR}_{\max }$ and BMI were unaffected.

\section{Discussion}

The present investigation has evaluated the effects of HIIT and CT in apparently healthy middle-aged adults. Our results show that HIIT performed at $94 \% \mathrm{HR}_{\text {max }}$ elicited a greater increase in $\dot{V} \mathrm{O}_{2 \max }, \dot{V} \mathrm{O}_{2}$ at VAT and $\dot{V} \mathrm{O}_{2} /$ HR when compared to $\mathrm{CT}\left(76 \% \mathrm{HR}_{\max }\right)$, despite $60 \%$ less training duration. Further, intervention fidelity was high in both interventions demonstrating excellent delivery of HIIT and CT, as well as its receipt and enactment by the participants.

Our results support previous research highlighting a greater increase in $\dot{V} \mathrm{O}_{2 \max }$ following 1:1 work/rest ratio HIIT, even when performed at lower weekly doses compared to existing literature $[14,16,36]$. As such the results highlight the role of intensity as an important factor to induce CRF adaptation [9]. In contrast, previous studies and meta-analyses have shown that low-volume HIIT is not superior than MICT in improving $\dot{V} \mathrm{O}_{2 \text { max }}$, eliciting similar changes [13, 15, 22, 37, 38]. However, these findings may be explained by the heterogeneous nature of the study populations and HIIT protocols prescribed. Our results are in agreement with previous studies which indicate a total of $64 \mathrm{~min}$ per week of MICT may be insufficient to provoke adaptions [39]. However, previous findings have shown increases in $\dot{V} \mathrm{O}_{2 \max }$ following $\mathrm{CT}$ when performed at higher doses [8, 20]. Thus, the trivial improvement in $\dot{V} \mathrm{O}_{2 \max }$ in the $\mathrm{CT}$ group may be due to the small volume of CT prescribed.

The increase in $\dot{V} \mathrm{O}_{2 \max }$ may be of clinical relevance given that $\dot{V} \mathrm{O}_{2 \max }$ is a strong prognostic marker of 

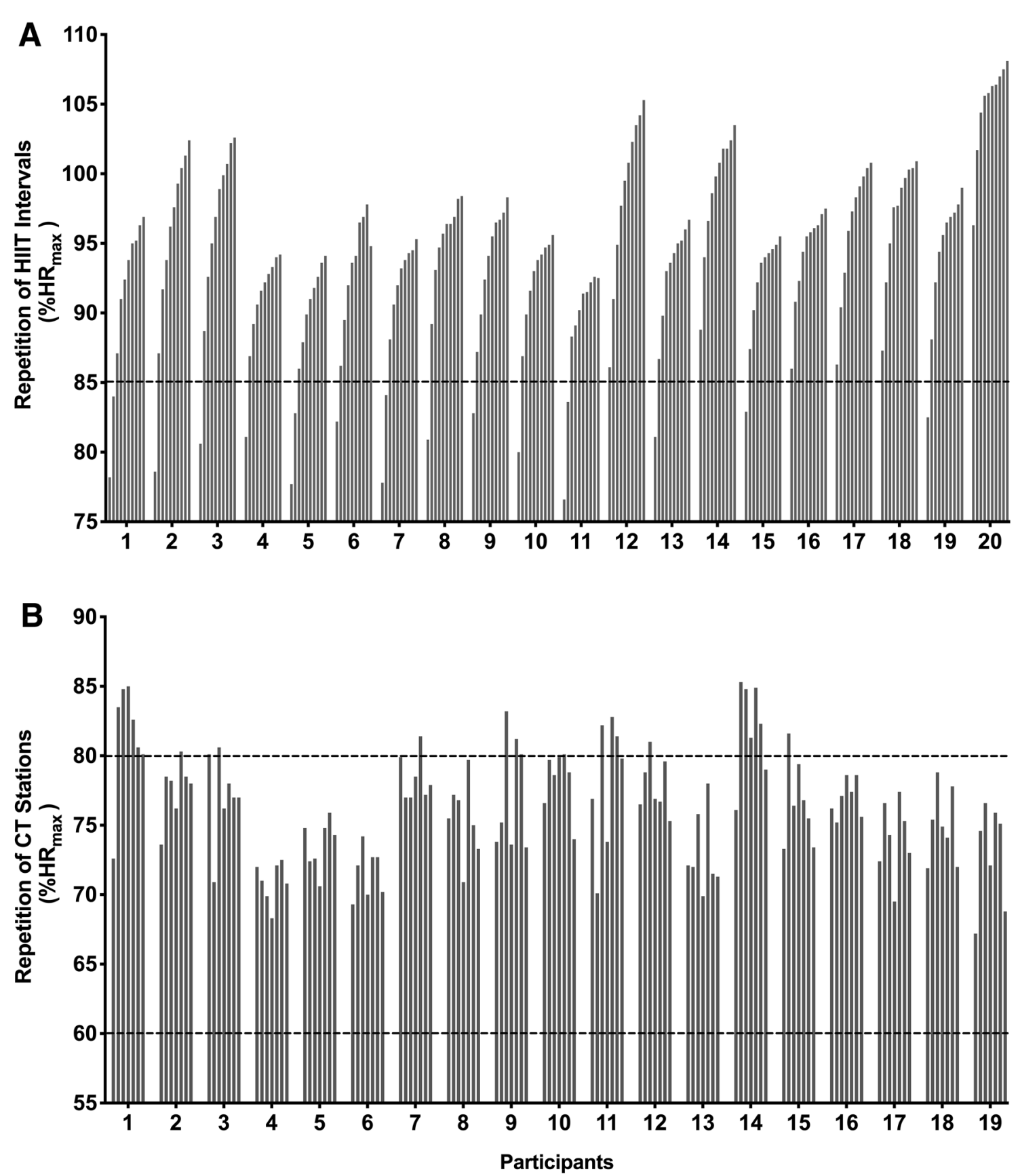

Fig. 1 Intervention fidelity. HIIT (a) and CT (b) mean heart rates during each interval or station of the interventions. Error bars have been omitted for figure clarity

all-cause and cardiovascular mortality and improving $\dot{V} \mathrm{O}_{2 \max }$ is associated with a reduction in mortality and cardiometabolic risk [3, 40]. Indeed, for every $3.5 \mathrm{ml} \mathrm{kg}^{-1} \mathrm{~min}^{-1}$ increase in $\dot{V} \mathrm{O}_{2 \max }$ there has been shown an $8-35 \%$ reduction in all-cause mortality and cardiovascular disease risk [3, 41]. As such, our findings suggest that HIIT may be a time-efficient and efficacious approach at inducing clinical meaningful benefits when compared to other popular training methods.
The present study showed that HIIT alone, significantly increased $\dot{V} \mathrm{O}_{2}$ at VAT, which is in agreement with previous studies [18]. In contrast, CT has been shown to increase the lactate threshold [17] when performed at higher doses, while a similar low-volume HIIT protocol to the current study reported improvements in $\dot{V} \mathrm{O}_{2}$ at VAT following both HIIT and MICT [37]. While this was time efficient, the relative training intensities of both groups were similar. Thus, the improvements in $\dot{V} \mathrm{O}_{2}$ at VAT in the present study are 

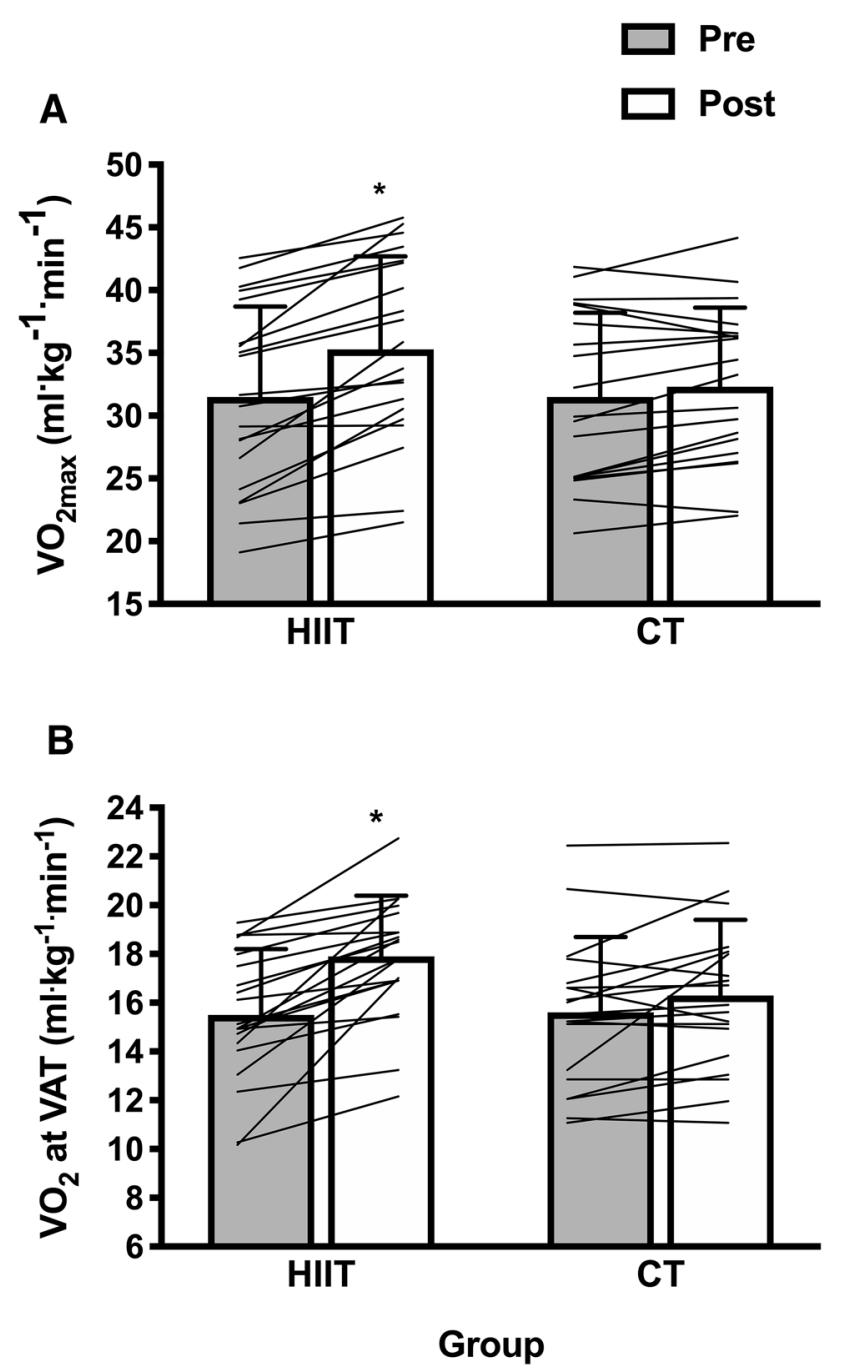

Fig. 2 Maximal oxygen consumption $\left(\dot{V} \mathrm{O}_{2 \max }\right)(\mathbf{a})$ and VAT (b) $\Delta$ after 8 weeks of HIIT $(n=20)$ and CT $(n=19)$. Black lines correspond to individual responses. $\dot{V} \mathrm{O}_{2}$ oxygen uptake, VAT ventilatory anaerobic threshold, HIIT low-volume high-intensity interval training, $C T$ circuit training. $* P<0.001$ versus baseline

likely due to the greater stimulus provided by HIIT. As such, HIIT consisting of two weekly sessions may be sufficient at inducing metabolic adaptations in skeletal muscle, enabling higher doses of exercise to be performed before lactate accumulation, thus becoming 'physiologically efficient' [42].

In agreement with previous research [16] our results showed that HIIT increased maximum $\dot{V} \mathrm{O}_{2} / \mathrm{HR}$ to a greater extent than CT, a surrogate marker of stroke volume (SV) and arteriovenous $\mathrm{O}_{2}$ difference. This is in contrast to recent findings [15]. Increases in maximum $\dot{V} \mathrm{O}_{2} / \mathrm{HR}$ are strongly correlated to an increase in SV [43]. While we did not directly measure SV or analyse muscle tissue our findings may support previous data which indicated lowvolume HIIT to be an optimal approach for inducing cardiovascular adaptations [44]. Studies adopting a similar low-volume HIIT protocol to the current study have shown increases in mitochondrial oxidative capacity $[45,46]$, while lower and higher volumes of HIIT have shown to be superior or comparable to MICT at increasing cardiac output and SV [47, 48]. While this is a positive finding from our results, further studies are required to confirm the mechanisms associated with an improvement in $\dot{V} \mathrm{O}_{2 \max }$ following a low-volume HIIT protocol consisting of a 1:1 work/rest ratio.

As well as reporting the attendance and mean exercise data, assessing if participants have performed the exercise training as intended is crucial when evaluating exercise interventions. Indeed, in the absence of evaluating intervention fidelity, incorrect conclusions, positive or negative, may be drawn about the effect of HIIT on a given outcome [49]. Using the pre-specified cut points for exercise intensity, $90 \%$ of the HIIT intervals and $86 \%$ of the CT stations met the criterion, indicating high fidelity to the interventions. Previous HIIT studies have reported low-to-moderate fidelity with compliance ranging between 23 and $63 \%$ [32, 34, 50]. The high levels of fidelity in the present study may be attributed to a number of factors. Firstly, the participants were healthy adults, they were made aware of their target HR each session and were given verbal encouragement to reach these targets. Secondly, the chosen HIT protocol in the present study may be more practical and less physically demanding [51]. High-volume HIIT protocols, or low-volume HIIT protocols involving 'all out' maximal effort (sprint interval training) may cause psychobiological stress, meaning that high levels of motivation are required to complete the protocol [52].

The failure to report fidelity, and the general inadequate reporting of exercise interventions is a major concern. With exercise intensity typically reported as a ranged based or as a general mean, this has inherent limitations. The authors strongly advocate that the CERT checklist [24] be adopted for future exercise studies as it provides reporting standards that ensures quality and transparency. As a result, exercise interventions may be clearly interpreted, translated, and implemented into practice.

There are a number of limitations that should be considered regarding this study. Not all participants achieved a $\dot{V} \mathrm{O}_{2}$ plateau during CPET as such secondary criteria was used which has established limitations. In addition, participants were not habituated to the CPET or the exercise sessions prior to starting the interventions. This may be relevant to novice or nervous participants in which familiarisation may be beneficial to obtain accurate measures. However, the use of strict criteria (i.e., a $\dot{V} \mathrm{O}_{2}$ plateau and heart rate) was met. Furthermore, laboratory-based studies investigating HIIT and MICT typically adopt the same modality of exercise and a similar or higher volume of training. While this is relevant regarding specificity of training, our aim was to assess two different low-volume training methods. 
Finally, for safety and accuracy of exercise prescription a maximal CPET was performed by all participants. However, access to specialised equipment may not always be feasible which may limit HIIT prescription in a "real world" setting. However, the present HIIT protocol appears well tolerated and safe, therefore further studies are required to evaluate the feasibility to adopt this protocol without a prior maximal CPET in healthy adults.

\section{Conclusion}

Our study demonstrated that HIIT is superior to CT, inducing larger improvements in CRF in apparently healthy middle-aged adults. Moreover, the present data provide evidence that 40 min per week of HIIT is sufficient to elicit clinical meaningful increases in $\dot{V} \mathrm{O}_{2}$. Furthermore, 64 min of CT per week appears not to provide sufficient stimulus to evoke CRF adaptation in this population. More broadly, this study provides further indication that HIIT and CT are a practical and effective approach to exercise training in healthy middle-aged adults given the high fidelity.

\section{Compliance with ethical standards}

Conflict of interest The authors declare that they have no competing interests.

Ethical approval Ethical approval for the study was given by the School of Life Sciences ethics committee at the University of Hull.

Informed consent Written consent was obtained by all.

Open Access This article is distributed under the terms of the Creative Commons Attribution 4.0 International License (http://creativeco mmons.org/licenses/by/4.0/), which permits unrestricted use, distribution, and reproduction in any medium, provided you give appropriate credit to the original author(s) and the source, provide a link to the Creative Commons license, and indicate if changes were made.

\section{References}

1. Blair SN, Morris JN (2009) Healthy hearts—and the universal benefits of being physically active: physical activity and health. Ann Epidemiol 19(4):253-256. https://doi.org/10.1016/j.annep idem.2009.01.019

2. Blair SN, Kohl HW 3rd, Paffenbarger RS Jr, Clark DG, Cooper KH, Gibbons LW (1989) Physical fitness and all-cause mortality. A prospective study of healthy men and women. JAMA 262(17):2395-2401

3. Ross R, Blair SN, Arena R, Church TS, Despres JP, Franklin BA, Haskell WL, Kaminsky LA, Levine BD, Lavie CJ, Myers J, Niebauer J, Sallis R, Sawada SS, Sui X, Wisloff U (2016) Importance of assessing cardiorespiratory fitness in clinical practice: a case for fitness as a clinical vital sign: a scientific statement from the american heart association. Circulation 134(24):e653-e699. https ://doi.org/10.1161/cir.0000000000000461

4. Lee D-C, Artero EG, Sui X, Blair SN (2010) Mortality trends in the general population: the importance of cardiorespiratory fitness. J Psychopharmacol 24(4_supplement):27-35. https://doi. org/10.1177/1359786810382057 (Oxford, England)

5. Kaminsky LA, Arena R, Beckie TM, Brubaker PH, Church TS, Forman DE, Franklin BA, Gulati M, Lavie CJ, Myers J, Patel MJ, Pina IL, Weintraub WS, Williams MA (2013) The importance of cardiorespiratory fitness in the United States: the need for a national registry: a policy statement from the American Heart Association. Circulation 127(5):652-662. https://doi.org/10.1161/ CIR.0b013e31827ee100

6. Thompson WR (2018) Worldwide survey of fitness trends for 2019. ACSM's Health Fit J 22(6):10-17. https://doi.org/10.1249/ fit.0000000000000438

7. Paoli A, Pacelli F, Bargossi AM, Marcolin G, Guzzinati S, Neri M, Bianco A, Palma A (2010) Effects of three distinct protocols of fitness training on body composition, strength and blood lactate. J Sports Med Phys Fit 50(1):43-51

8. Myers TR, Schneider MG, Schmale MS, Hazell TJ (2015) Wholebody aerobic resistance training circuit improves aerobic fitness and muscle strength in sedentary young females. J Strength Cond Res 29(6):1592-1600. https://doi.org/10.1519/jsc.0000000000 000790

9. Gibala MJ, Little JP, Macdonald MJ, Hawley JA (2012) Physiological adaptations to low-volume, high-intensity interval training in health and disease. J Physiol 590(Pt 5):1077-1084. https:// doi.org/10.1113/jphysiol.2011.224725

10. Weston KS, Wisløff U, Coombes JS (2014) High-intensity interval training in patients with lifestyle-induced cardiometabolic disease: a systematic review and meta-analysis. Br J Sports Med 48(16):1227-1234. https://doi.org/10.1136/bjsports-2013-092576

11. Paoli A, Pacelli QF, Moro T, Marcolin G, Neri M, Battaglia G, Sergi G, Bolzetta F, Bianco A (2013) Effects of high-intensity circuit training, low-intensity circuit training and endurance training on blood pressure and lipoproteins in middle-aged overweight men. Lipids Health Dis 12(1):131. https://doi. org/10.1186/1476-511x-12-131

12. Weston KS, Wisløff U, Coombes JS (2014) High-intensity interval training in patients with lifestyle-induced cardiometabolic disease: a systematic review and meta-analysis. Br J Sports Med 48(16):1227-1234 1228p. https://doi.org/10.1136/bjsports-2013092576

13. Sawyer BJ, Tucker WJ, Bhammar DM, Ryder JR, Sweazea KL, Gaesser GA (2016) Effects of high-intensity interval training and moderate-intensity continuous training on endothelial function and cardiometabolic risk markers in obese adults. J Appl Physiol 121(1):279-288. https://doi.org/10.1152/japplphysiol.00024.2016

14. Esfandiari S, Sasson Z, Goodman JM (2014) Short-term highintensity interval and continuous moderate-intensity training improve maximal aerobic power and diastolic filling during exercise. Eur J Appl Physiol 114(2):331-343. https://doi.org/10.1007/ s00421-013-2773-x

15. Baekkerud FH, Solberg F, Leinan IM, Wisloff U, Karlsen T, Rognmo O (2016) Comparison of three popular exercise modalities on $V \mathrm{O}_{2 \max }$ in overweight and obese. Med Sci Sports Exerc 48(3):491-498. https://doi.org/10.1249/mss.0000000000000777

16. Boyd JC, Simpson CA, Jung ME, Gurd BJ (2013) Reducing the intensity and volume of interval training diminishes cardiovascular adaptation but not mitochondrial biogenesis in overweight/ obese men. PLoS One 8(7):e68091. https://doi.org/10.1371/journ al.pone. 0068091

17. Takeshima N, Rogers ME, Islam MM, Yamauchi T, Watanabe E, Okada A (2004) Effect of concurrent aerobic and resistance circuit 
exercise training on fitness in older adults. Eur J Appl Physiol 93(1-2):173-182. https://doi.org/10.1007/s00421-004-1193-3

18. Ziemann E, Grzywacz T, Luszczyk M, Laskowski R, Olek RA, Gibson AL (2011) Aerobic and anaerobic changes with highintensity interval training in active college-aged men. J Strength Cond Res 25(4):1104-1112. https://doi.org/10.1519/JSC.0b013 e3181d09ec9

19. Matsuo T, Saotome K, Seino S, Eto M, Shimojo N, Matsushita A, Iemitsu M, Ohshima H, Tanaka K, Mukai C (2014) Lowvolume, high-intensity, aerobic interval exercise for sedentary adults: \$\$ldot $\{\mathrm{V}\} \$$ O O, cardiac mass, and heart rate recovery. Eur J Appl Physiol 114(9):1963-1972

20. McRae G, Payne A, Zelt JGE, Scribbans TD, Jung ME, Little JP, Gurd BJ (2012) Extremely low volume, whole-body aerobicresistance training improves aerobic fitness and muscular endurance in females. Appl Physiol Nutr Metab 37(6):1124-1131. https://doi.org/10.1139/h2012-093

21. McGregor G, Nichols S, Hamborg T, Bryning L, Tudor-Edwards R, Markland D, Mercer J, Birkett S, Ennis S, Powell R, Begg B, Haykowsky MJ, Banerjee P, Ingle L, Shave R, Backx K (2016) High-intensity interval training versus moderate-intensity steady-state training in UK cardiac rehabilitation programmes (HIIT or MISS UK): study protocol for a multicentre randomised controlled trial and economic evaluation. BMJ Open 6(11):e012843. https://doi.org/10.1136/bmjopen-2016-012843

22. Weston M, Taylor K, Batterham A, Hopkins W (2014) Effects of low-volume high-intensity interval training (HIT) on fitness in adults: a meta-analysis of controlled and non-controlled trials. Sports Med 44(7):1005-1017. https://doi.org/10.1007/s4027 9-014-0180-z

23. Nordestgaard BG, Chapman MJ, Ray K, Boren J, Andreotti F, Watts GF, Ginsberg H, Amarenco P, Catapano A, Descamps OS, Fisher E, Kovanen PT, Kuivenhoven JA, Lesnik P, Masana L, Reiner Z, Taskinen MR, Tokgozoglu L, Tybjaerg-Hansen A, European Atherosclerosis Society Consensus P (2010) Lipoprotein(a) as a cardiovascular risk factor: current status. Eur Heart J 31(23):2844-2853. https://doi.org/10.1093/eurhe artj/ehq386

24. Slade SC, Dionne CE, Underwood M, Buchbinder R (2016) Consensus on exercise reporting template (CERT): explanation and elaboration statement. Br J Sports Med 50:1428-1437

25. Bolgar MR, Baker CE, Goss FL, Nagle E, Robertson RJ (2010) Effect of exercise intensity on differentiated and undifferentiated ratings of perceived exertion during cycle and treadmill exercise in recreationally active and trained women. J Sports Sci Med 9(4):557-563

26. Ross RM (2003) ATS/ACCP statement on cardiopulmonary exercise testing. Am J Respir Crit Care Med 167(10):1451. https ://doi.org/10.1164/ajrccm.167.10.950 (author reply 1451)

27. Balady GJ, Arena R, Sietsema K, Myers J, Coke L, Fletcher GF, Forman D, Franklin B, Guazzi M, Gulati M, Keteyian SJ, Lavie CJ, Macko R, Mancini D, Milani RV, American Heart Association Exercise CR, Prevention Committee of the Council on Clinical C, Council on E, Prevention, Council on Peripheral Vascular D, Interdisciplinary Council on Quality of C, Outcomes R (2010) Clinician's guide to cardiopulmonary exercise testing in adults: a scientific statement from the American Heart Association. Circulation 122(2):191-225. https://doi. org/10.1161/cir.0b013e3181e52e69

28. Wasserman K, Hansen J, Sue D, Stringer W, Sietsema K, Sun X-G, Whipp B (2011) Principles of exercise testing and interpretation: including pathophysiology and clinical applications, 5th edn. Lippincott Williams \& Wilkins, Philadelphia

29. Borg GA (1982) Psychophysical bases of perceived exertion. Med Sci Sports Exerc 14(5):377-881
30. Astorino TA (2009) Alterations in VOmax and the VO plateau with manipulation of sampling interval. Clin Physiol Funct Imaging 29(1):60-67. https://doi.org/10.1111/j.1475097X.2008.00835.x

31. Beaver WLWK, Whipp BJ (1986) A new method for detecting anaerobic threshold by gas exchange. J Appl Physiol 60:2020-2027

32. Taylor KL, Weston M, Batterham AM (2015) Evaluating intervention fidelity: an example from a high-intensity interval training study. PLoS One 10(4):e0125166. https://doi.org/10.1371/ journal.pone.0125166

33. Weston M, Batterham AM, Tew GA, Kothmann E, Kerr K, Nawaz S, Yates D, Danjoux G (2017) Patients awaiting surgical repair for large abdominal aortic aneurysms can exercise at moderate to hard intensities with a low risk of adverse events. Front Physiol 7:684. https://doi.org/10.3389/fphys.2016.00684

34. Hurst C, Weston KL, Weston M (2018) The effect of 12 weeks of combined upper- and lower-body high-intensity interval training on muscular and cardiorespiratory fitness in older adults. Aging Clin Exp Res. https://doi.org/10.1007/s40520-018-1015-9

35. Richardson JTE (2011) Eta squared and partial eta squared as measures of effect size in educational research. Educ Res Rev 6(2):135-147. https://doi.org/10.1016/j.edurev.2010.12.001

36. Stavrinou PS, Bogdanis GC, Giannaki CD, Terzis G, Hadjicharalambous M (2018) High-intensity interval training frequency: cardiometabolic effects and quality of life. Int J Sports Med 39(3):210-217. https://doi.org/10.1055/s-0043-125074

37. Currie KD, Dubberley JB, McKelvie RS, MacDonald MJ (2013) Low-volume, high-intensity interval training in patients with CAD. Med Sci Sports Exerc 45(8):1436-1442. https://doi. org/10.1249/MSS.0b013e31828bbbd4

38. McKay BR, Paterson DH, Kowalchuk JM (2009) Effect of shortterm high-intensity interval training vs continuous training on $\mathrm{O}_{2}$ uptake kinetics, muscle deoxygenation, and exercise performance. J Appl Physiol 107(1):128-138. https://doi.org/10.1152/jappl physiol.90828.2008

39. Montero D, Lundby C (2017) Refuting the myth of non-response to exercise training: 'non-responders' do respond to higher dose of training. J Physiol 595(11):3377-3387. https://doi.org/10.1113/ jp273480

40. Ingle L, Mellis M, Brodie D, Sandercock GR (2016) Associations between cardiorespiratory fitness and the metabolic syndrome in British men. Heart. https://doi.org/10.1136/heartjnl-2016-31014

41. Kodama S, Saito K, Tanaka S, Maki M, Yachi Y, Asumi M, Sugawara A, Totsuka K, Shimano H, Ohashi Y, Yamada N, Sone H (2009) Cardiorespiratory fitness as a quantitative predictor of all-cause mortality and cardiovascular events in healthy men and women: a meta-analysis. JAMA 301(19):2024-2035. https://doi. org/10.1001/jama.2009.681

42. Ghosh AK (2004) Anaerobic threshold: its concept and role in endurance sport. Malays J Med Sci 11(1):24-36

43. Bhambhani Y, Norris S, Bell G (1994) Prediction of stroke volume from oxygen pulse measurements in untrained and trained men. Can J Appl Physiol 19(1):49-59

44. Daussin FN, Ponsot E, Dufour SP, Lonsdorfer-Wolf E, Doutreleau S, Geny B, Piquard F, Richard R (2007) Improvement of $\mathrm{VO}_{2 \max }$ by cardiac output and oxygen extraction adaptation during intermittent versus continuous endurance training. Eur J Appl Physiol 101(3):377-383. https://doi.org/10.1007/s00421-007-0499-3

45. Hood MS, Little JP, Tarnopolsky MA, Myslik F, Gibala MJ (2011) Low-volume interval training improves muscle oxidative capacity in sedentary adults. Med Sci Sports Exerc 43(10):1849-1856. https://doi.org/10.1249/MSS.0b013e3182199834

46. Little JP, Safdar A, Wilkin GP, Tarnopolsky MA, Gibala MJ (2010) A practical model of low-volume high-intensity interval 
training induces mitochondrial biogenesis in human skeletal muscle: potential mechanisms. J Physiol 588(Pt 6):1011-1022. https ://doi.org/10.1113/jphysiol.2009.181743

47. Astorino TA, Edmunds RM, Clark A, King L, Gallant RM, Namm S, Fischer A, Wood KA (2016) High-intensity interval training increases cardiac output and $\mathrm{VO}_{2 \max }$. Med Sci Sports Exerc 5:6656. https://doi.org/10.1249/mss.0000000000001099

48. Helgerud J, Hoydal K, Wang E, Karlsen T, Berg P, Bjerkaas M, Simonsen T, Helgesen C, Hjorth N, Bach R, Hoff J (2007) Aerobic high-intensity intervals improve $\mathrm{VO}_{2 \max }$ more than moderate training. Med Sci Sports Exerc 39(4):665-671. https://doi. org/10.1249/mss.0b013e3180304570

49. Fortington LV, Donaldson A, Lathlean T, Young WB, Gabbe BJ, Lloyd D, Finch CF (2015) When 'just doing it' is not enough: assessing the fidelity of player performance of an injury prevention exercise program. J Sci Med Sport 18(3):272-277. https:// doi.org/10.1016/j.jsams.2014.05.001

50. Weston M, Batterham AM, Tew GA, Kothmann E, Kerr K, Nawaz S, Yates D, Danjoux G (2016) Patients awaiting surgical repair for large abdominal aortic aneurysms can exercise at moderate to hard intensities with a low risk of adverse events. Front Physiol 7:684. https://doi.org/10.3389/fphys.2016.00684

51. Gibala MJ, Little JP, Macdonald MJ, Hawley JA (2012) Physiological adaptations to low-volume, high-intensity interval training in health and disease. J Physiol 590(5):1077-1084. https://doi. org/10.1113/jphysiol.2011.224725

52. Hardcastle SJ, Ray H, Beale L, Hagger MS (2014) Why sprint interval training is inappropriate for a largely sedentary population. Front Psychol 5:1505. https://doi.org/10.3389/fpsyg .2014 .01505

Publisher's Note Springer Nature remains neutral with regard to jurisdictional claims in published maps and institutional affiliations. 